\title{
Two-Section Gain-Levered Tunable Distributed Feedback Laser with Active Tuning Section
}

\author{
Giora Griffel, Robert J. Lang, and Amnon Yariv, Fellow, IEEE
}

\begin{abstract}
A composite cavity semiconductor laser structure that consists of two active (gain) sections is analyzed. One of the sections has a grating corrugation, while the other is flat. The operation of the device is enhanced by utilizing the gain-lever effect. A round-trip analysis is combined with the rate equations pertinent for this structure to study the characteristics of the device. The performance of the device is compared to that of a similar case without the gain lever. It is shown that, utilizing the gain-lever effect, improved operation in terms of tuning range, modulation efficiency, and output power control can be obtained.
\end{abstract}

\section{INTRODUCTION}

A $S$ the interest in fast, tunable (FM) light sources has grown, it became clear that due to the shorter photon lifetime under stimulated emission, the tuning section of a tunable multisection laser diode should be active rather than passive [1], [2]. The superiority of an active tuning section over other schemes of direct modulation became even more apparent with the utilization of the gain-lever effect [3]-[5], which can enhance the modulation response and widen the tuning range. However, the design and analysis of such highspeed, multiple-active-section laser diodes are complicated by the strong interaction between all the sections in determining the operating condition. The threshold condition, for example, is determined in this case by an infinite number of possible carrier density combinations of the different gain sections.

In this paper, we analyze a two-section semiconductor laser structure. The two sections are active and can control the optical wavelength, the output power, and the modulation response. In addition, the gain-lever effect is utilized to improve the device operation by biasing the two sections at different points along the gain-carrier density curve. Due to the nonlinearity of the gain-carrier density curve, a small modulation of the carrier density in the section biased close to transparency results in a large compensating change in the other section [3]. One of the two sections has a distributed feedback structure, that is, a corrugation grating is formed over the active layer. The other section is also active and contributes to the overall gain, but has no corrugation. The approach that we present for the analysis of this device is comprehensive and

Manuscript received April 14, 1993. This work was supported in part by the Engineering Foundation IEEE Life Member Fund and in part by a grant from the New York State Science and Technology Foundation.

G. Griffel is with the Center for Advanced Technology in Telecommunication, Polytechnic University of New York, Brooklyn, NY 11201.

R. J. Lang is with the Spectra Diode Laboratories, San Jose, CA 95134.

A. Yariv is with the Department of Applied Physics, Califomia Institute of Technology, Pasadena, CA 91125.

IEEE Log Number 9214955. can be used to treat other multisection, tunable diode lasers with active tuning section.

A round-trip analysis for the composite cavity structure is used to obtain the exact operating condition, the tuning behavior, and the optical power-current relationship. For the case of a two-gain-section device, this results in a complex secular equation with three independent variables-the carrier concentrations in each section, and the lasing frequency. An iterated root searching technique is then used to solve the associated secular equation and to calculate and plot the threshold condition for all the possible longitudinal modes. Using these threshold lines, along with the carrier rate equations and the relation between the photon density in each section and the output power, we have calculated the wavelength and optical output power for any possible combination of the electrode currents [6]. The results are used to compare the performance of a regular and gain-levered mode of operation in terms of tuning range and modulation response.

\section{FORMULATION}

The device that we have analyzed is depicted schematically in Fig. 1. Here, two sections are active, and thus both contribute to the overall gain. However, only one of the gain sections is made frequency-selective by forming a reflection grating over the active region. We begin our analysis by deriving the eigenvalue equation of the lasing modes. We do so by considering the two complex amplitude reflections $\rho_{1,2}$ from the two segments to the left and to the right of the dividing line shown in Fig. 1. We adopt the time convention $\exp (i \omega t)$. The reflectivity $\rho_{1}$ seen looking to the left is, by inspection,

$$
\rho_{1}=r \exp \left[-i 2 h L \gamma_{1}\right]
$$

where $r$ is the facet reflectivity, $h$ is the fraction of the cavity taken up by the noncorrugated section, $L$ is the overall length, and $\gamma_{1}$ is the complex propagation constant defined by

$$
\gamma_{1}=\frac{2 \phi}{\lambda} n_{\mathrm{eff}_{1}}+\frac{g\left(N_{1}\right)}{2}\left(-\alpha_{1}+i\right) .
$$

In (2), $n_{\mathrm{eff}_{1}}$ is the effective refractive index of the mode supported by the cavity, $g\left(N_{1}\right)$ is the sublinear gain-carrier relation responsible for the gain-lever effect, and $\alpha_{1}$ is the linewidth enhancement factor in section 1 , which is defined by

$$
\alpha_{1} \equiv\left(\frac{\Delta n_{r} / \Delta N}{\Delta n_{i} / \Delta N}\right)_{1}
$$




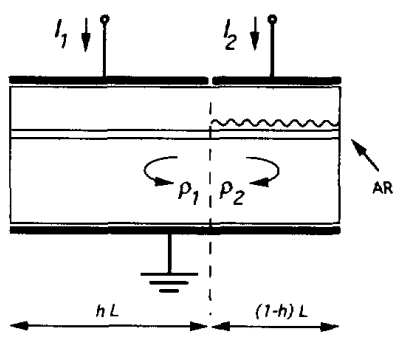

Fig. 1. Schematic structure of two-active section semiconductor laser.

where $\Delta n_{r}$ and $\Delta n_{i}$ are the changes in the real and imaginary part of the complex refractive index due to a change $\Delta N$ in the carrier density. $\alpha_{2}$ is similarly defined for section 2 . The mode wavelength shift due to the gain-lever effect can be described, to the first order, in terms of the linewidth enhancement factors of the two sections by [6]

$$
\Delta \lambda=\frac{\lambda^{2}}{4 \pi n_{g}}\left[h \alpha_{1}-(1-h) \alpha_{2}\right]
$$

where $n_{g}$ is the group refractive index. For the case of equal lengths of the two sections, the wavelength shift is proportional to the difference between the enhancement factors of the sections $\alpha_{1}-\alpha_{2}$. Therefore, a difference in $\alpha$ would result in a net change in the wavelength of operation. The fractional length of the sections can be devised to optimize the tuning range [5].

The reflectivity $\rho_{2}$ of the corrugated section seen looking to the right is given by [7]

$$
\rho_{2}=\frac{\kappa \sinh \left[s_{2}(1-h) L\right]}{t_{2} \sinh \left[s_{2}(1-h) L\right]-s_{2} \cosh \left[s_{2}(1-h) L\right]}
$$

where $\kappa$ is the grating coupling coefficient and $s_{2}$ is a parameter defined by

$$
s_{2}=\left\{\kappa^{2}+\left[\frac{g\left(N_{2}\right)}{2}-i \Delta \beta_{2}\right]^{2}\right\}^{1 / 2} \equiv\left[\kappa^{2}+t_{2}^{2}\right]^{1 / 2} .
$$

In (6), $\Delta \beta_{2}$ designates the detuning from the Bragg condition that takes the carriers' influence into consideration. The lasing condition $\rho_{1} \cdot \rho_{2}=1$ yields the secular equation

$$
\left[r \exp \left(-i 2 h L \gamma_{1}\right)-t_{2}\right] \tanh \left[s_{2}(1-h) L\right]=s_{2} .
$$

Equation (7) is a complex equation with three unknowns- $N_{1}$ and $N_{2}$, the carrier concentrations in the two sections, and $\omega$, the lasing frequency. Any particular steady-state solution consists of a set of these three variables, each having a real value. Equation (6) was solved numerically using iterated root searching and curve tracing techniques [6]. This result is shown in Fig. 2 as a family of lines in the $N_{1} / N_{2}$ plane. Each of these lines corresponds to a different longitudinal mode. Due to gain clamping, only values of $\left(N_{1}, N_{2}\right)$ that lie on one of these lines are allowed steady-state operating conditions of a specific longitudinal mode. The third variable $\omega$ is not shown in this plot. Therefore, one

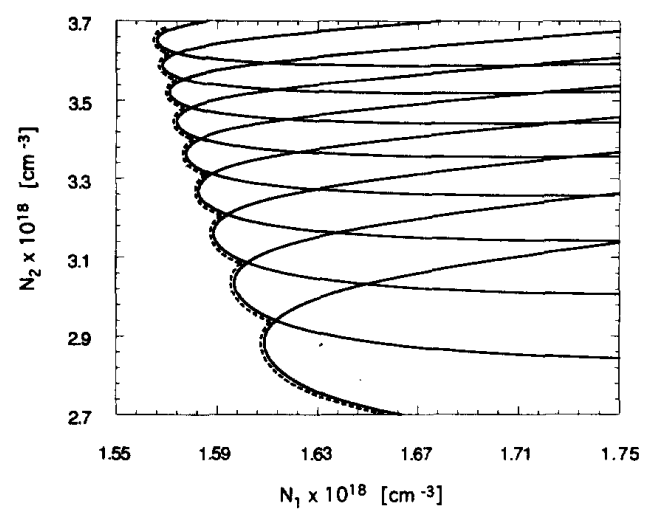

Fig. 2. Cutoff lines of nine consecutive longitudinal modes. The carrier concentration clamping line is shown by the dashed line.

should look at this plot as a projection of the cutoff lines on the $N_{1} / N_{2}$ plane, where each point along the curve has a different lasing frequency. A detailed discussion of a similar analysis for the case of the longitudinally coupled-cavity laser is given in [8]. For the calculation, we assumed a two-section device with a total length of $470 \mu \mathrm{m}$, and fractional lengths of 0.7 and 0.3 for the noncorrugated section and the corrugated section, respectively. For the gain-carrier dependency, we have used a sublinear relation with a transparency carrier density of $1.4 \times 10^{18} \mathrm{~cm}^{-3}$. The noncorrugated section is biased close to transparency, while the other section is biased for high gain to provide the lasing condition.

Due to the spontaneous emission in both sections, the carrier densities of the laser are clamped just to the left of the line which is comprised of the lowest segments of the different longitudinal-mode cutoff lines, as shown in Fig. 2. Changing the operating conditions of the laser is performed by varying the driving currents that, in turn, will vary the carrier concentrations. In this case, the laser operating point is traveling along the cutoff line. Walking along the segments between the cusps corresponds to a continuous frequency turning, while passing from one segment to the neighboring one through a cusp corresponds to a mode hop.

To obtain the tuning curves and the light-current relations, we have invoked the rate equations for the carriers in the two sections:

$$
\frac{d N_{i}}{d t}=\frac{j_{i}}{e d}-\frac{N_{i}}{\tau_{e}}-\Gamma \nu_{g_{i}} g\left(N_{i}\right) p_{i}
$$

where $e$ is the electron charge, $d$ is the thickness of the active layer, $\nu_{g}$ is the group velocity, $n_{i}$ indicates the carrier density in each section, $j_{i}$ is the current density in the $i$ th section, where $i=1,2$, and $p_{i}$ is the photon density. The parameter $\Gamma$ is the fill factor, and $\tau_{e}$ is the carriers lifetime. In steady state, $d / d t=0$ and one can obtain the relation between the driving currents and the output power:

$$
I_{i}=e A_{i} \frac{N_{i}}{\tau_{e}}+e \Gamma \nu_{g_{i}} g\left(N_{i}\right) \frac{P_{\text {out }}}{\xi_{i}} .
$$

Here, $A_{i}$ is the section area, while the parameter $\xi_{i}$ represents the relation between the photon density in each section 


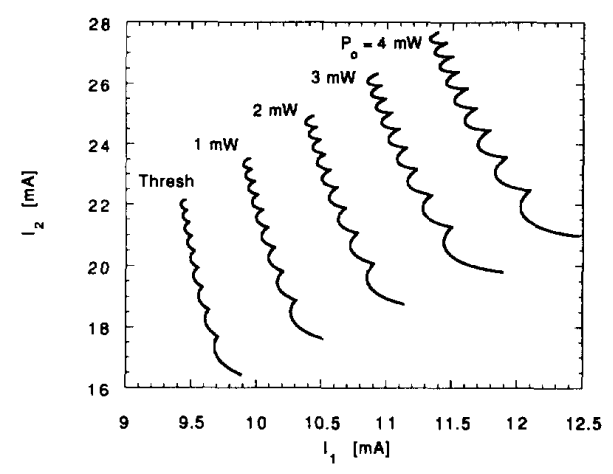

Fig. 3. Equipower lines in the current's $I_{1} / I_{2}$ plan.

and the output power emitted from the noncorrugated section facet [6]:

$$
\xi_{1}=E \nu_{g}(1-R) \exp \left\{\left[\Gamma g\left(N_{1}\right)-\alpha_{0}\right] h L\right\} / F_{1}
$$

and

$$
\xi_{2}=E \nu_{g} \frac{(1-R)}{R} \exp \left\{-\left[\Gamma g\left(N_{1}\right)-\alpha_{0}\right] h L\right\} / F_{2}
$$

where $E$ is the photon energy, $R$ is the mirror reflectivity at the facet of the noncorrugated section, $\alpha_{0}$ is the absorption loss, and $F_{1}$ and $F_{2}$ are normalized integrals of the square of the electric field amplitudes in sections 1 and 2 , respectively.

\section{RESULTS AND DISCUSSION}

We have used the described analysis to transfer the cutoff line into a mode map in the current $I_{1} / I_{2}$ plane, which is shown in Fig. 3. Here, we plot the relevant segments of the different longitudinal modes and connect those that correspond to a constant output power. The first term on the right-hand side of (9) provides the threshold current values, while the second term indicates the necessary increase of the section currents $\Delta I_{i}$ that is required to obtain a specific output power: For the case that we have analyzed, section 1 is biased close to transparency to utilize the gain-lever effect. As a result, the lines corresponding to different values of a constant output power are getting steeper as $I_{1}$ is reduced. This kind of mode map is a useful tool for establishing a specific mode of operation for the device. Variation of the driving currents in a proportional manner is manifested by a straight line in this plane. The most common practice would be, of course, to hold one of the currents at a fixed value while continuously sweeping the other section current. This would correspond to either a vertical or a horizontal line on this plane. It is also possible to form a linear relation between the driving currents. In this way, for example, one can tune the laser wavelength while maintaining an almost constant output power by moving along a straight line that is tangential to any of the constant-power lines in the mode map, as shown in Fig. 3.

In Fig. 4, we plot the tuning curve of this device where $I_{2}$, the corrugation section current, is held constant at 20 $\mathrm{mA}$, while $I_{1}$ is varied continuously close to its transparency value. It is shown here that the wavelength tuning curve has a

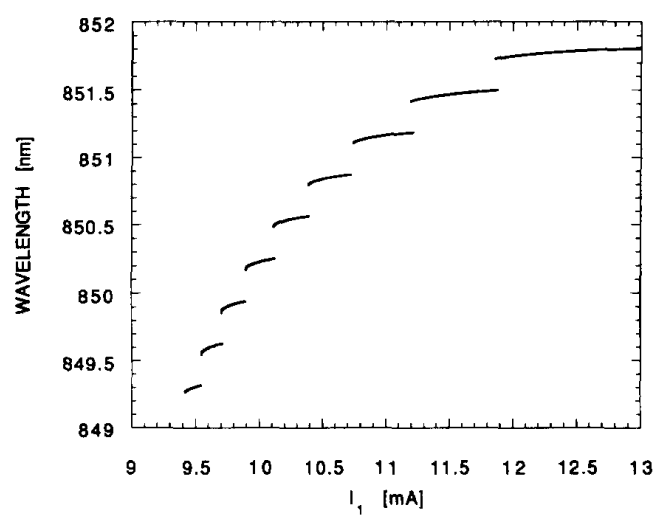

Fig. 4. Tuning curve as a function of the noncorrugated section current $I_{1}, I_{2}$ is held fixed at $20 \mathrm{~mA}$

typical "slews-and-hops" shape. One should note the gradual increase in the slope of the continuous segments, which means improved tunability, as $I_{1}$ approaches transparency; this behavior is due to the nonlinear gain-lever effect. The enhancement of the device operation is also observed by looking at the wavelength tuning range which is obtained by varying $I_{2}$ while $I_{1}$ is being held fixed and close to transparency. It requires over five times the amount of change in $I_{2}$, which is far from the transparency value, to obtain the same amount of wavelength change as achieved by holding $I_{2}$ fixed and varying $I_{1}$.

We have also carried out an identical analysis of the same device for the case where the carrier concentrations were nearly identical in both sections. In such a situation, no gain-lever effect persists. The characteristics of the tuning curves and the output power/current dependency are similar. However, the tunability in this case is almost two orders of magnitude less than in the gain levered case $(0.072 \mathrm{~nm} / \mathrm{mA}$ at the steepest slope compared to $4.8 \mathrm{~nm} / \mathrm{mA}$ in the gain-levered case). This result agrees well with previous predictions

Finally, we have calculated the dependency of the optical output power on the variation of the different electrode currents. Fig. 5 shows the output power as a function of the current in section 1 when the current in section 2 is fixed at 20 $\mathrm{mA}$. Note the close-to-linear relation, which is a close match to experimental results obtained previously for an InGaAsP-InP double-channel planar buried heterostructure laser with a very similar structure [9]. Another important feature is shown in Fig. 6 where the output power is plotted as a function of the current in section 2 . Here, we observe local maxima, that is, points of zero slope, one for each longitudinal mode. This behavior is caused by the strong frequency dependency of the distributed reflection from Bragg grating in section 2 . As the current in this section is changed, the frequency of the reflection peak is continuously moving. The longitudinal mode locations are also moving, but at a different rate. For each mode, there is a point of minimum loss that occurs when the longitudinal mode frequency coincides with the reflection peak. At that point, the fewest carriers are needed to provide the threshold condition, and the rest contribute to the output 


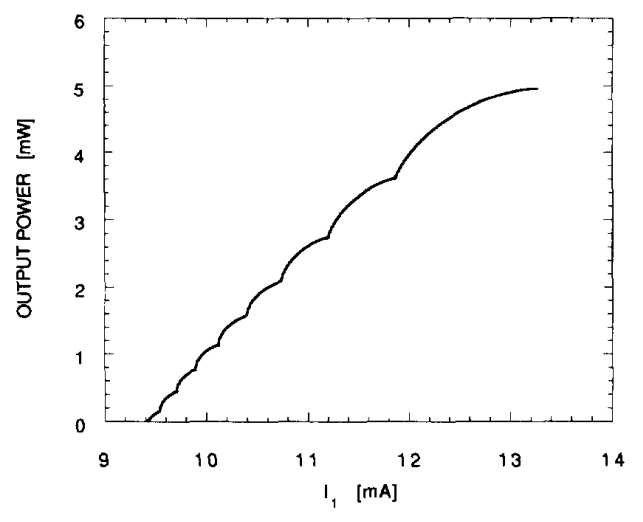

Fig. 5. Output power versus current in the noncorrugated section.

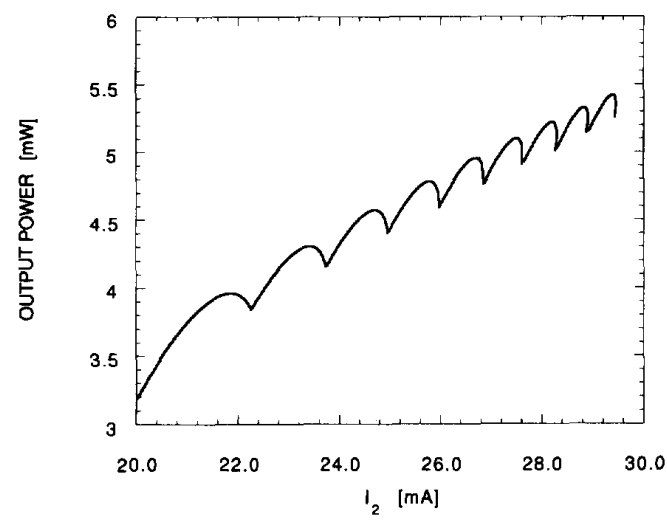

Fig. 6. Output power versus current in section 2. Note the local maxima for the different longitudinal modes.

power which attains its maximum. We can use this behavior to achieve improved FM/AM modulation ratio by biasing the current in section 2 to any of these points.

\section{CONCLUSION}

We proposed and analyzed a two-section tunable semiconductor laser with one corrugated section utilizing the gain-lever effect. We used the formulation described above to plot the cutoff lines, the mode map, the tuning curves, and the light/current dependency, as well as to optimize the device operation. The analysis is based on a round-trip formulation, along with the rate equation and light/carrier dependency of the specific device. We have shown that by using this structure while utilizing the gain-lever effect, improved tuning behavior and better control of the output power/current relations is feasible. The results are in good agreement with previous experimental results. The presented formulation is a valuable tool for reliable design and a better understanding of the operation of this family of high-speed tunable semiconductor lasers.

\section{REFERENCES}

[1] M. Kuznetzov, "Theory of wavelength tuning in two-segment distributed feedback lasers," IEEE J. Quantum Electron., vol. 24, pp. 1837-1844, 1988.

[2] T. L. Koch and U. Koren, "Semiconductor lasers for coherent optical fiber communications," J. Lightwave Technol., vol. 8, pp. 274-293, 1990.

[3] K. J. Vahala, M. A. Newkirk, and T. C. Chen, "The optical gain lever: A novel gain mechanism in the direct modulation of quantum well semiconductor lasers," Appl. Phys. Lett., vol. 54, pp. 2506-2508, 1989.

[4] K. Y. Lau, "Frequency modulation and linewidth of gain-levered twosection single quantum well lasers," Appl. Phys. Lett., vol. 57, pp. 2068-2070, 1990

[5] $\{-\}-\}$, "Broad wavelength tunability in gain-levered quantum well semiconductor lasers," Appl. Phys. Lett., vol. 57, pp. 2632-2634, 1990.

[6] G. Griffel, R. J. Lang, and A. Yariv, in preparation.

[7] A. Yariv, Optical Electronics, 4th ed. Philadelphia, PA: Saunders, 1991.

[8] R. J. Lang and A. Yariv, "Intermodal stability of a coupled-cavity semiconductor laser," IEEE J. Quantum Electronics, vol. QE-22, pp. 631-636, 1986

[9] B. Broberg and S. Nilsson, "Widely tunable active Bragg reflector integrated lasers in InGaAsP-InP," Appl. Phys. Lett., vol. 52, pp. $1285-1287,1988$

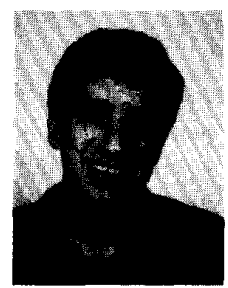

Giora Griffel was bom in Tel Aviv, Israel, in October 1955. He received the B.Sc. (Summa cum laude) in electrical engineering, and the $\mathrm{Ph} . \mathrm{D}$. degree from Tel Aviv University in 1982 and 1989 , respectively. From 1989 to 1990 he was a postdoctoral research fellow at the California Institute of Technology, Pasadena, CA. In 1991 he joined the Polytechnic University of New York as an assistant professor of electrical engineering. His current research interests include dynamic and spectral properties of composite-cavity and coupled-cavity semiconductor lasers, tunable semiconductor lasers, and guided-wave devices. He is a cochair of the 1994 Weber Research Institute International Symposium on Guided Wave Optoelectronics. He has been the author or coauthor of some 30 journal papers and a similar number of conference papers.

Dr. Griffel is a member of the Optical Society of America.

Robert J. Lang, photograph and biography not available at the time of publication.

Amnon Yariv, photograph and biography not available at the time of publication. 